\title{
Solution NMR structures reveal unique homodimer formation by a winged helix-turn-helix motif and provide first structures for protein domain family PF10771
}

\author{
Alexander Eletsky, \\ Department of Chemistry, The State University of New York at Buffalo, and Northeast Structural \\ Genomics Consortium, Buffalo, NY 14260, USA szypersk@buffalo.edu \\ Donald Petrey, \\ Department of Biochemistry and Molecular Biophysics, Howard Hughes Medical Institute, Center \\ for Computational Biology and Bioinformatics, Columbia University, New York, NY 10032, USA

\section{Qiangfeng Cliff Zhang,} \\ Department of Biochemistry and Molecular Biophysics, Howard Hughes Medical Institute, Center \\ for Computational Biology and Bioinformatics, Columbia University, New York, NY 10032, USA

\section{Hsiau-Wei Lee, \\ Complex Carbohydrate Research Center, University of Georgia, and Northeast Structural Genomics Consortium, Athens, GA 30602, USA}

\section{Thomas B. Acton,}

Department of Molecular Biology and Biochemistry, Center of Advanced Biotechnology and Medicine, Rutgers, The State University of New Jersey, Piscataway, NJ 08854, USA

Department of Biochemistry, Robert Wood Johnson Medical School, UMDNJ, and Northeast Structural Genomics Consortium, Piscataway, NJ 08854, USA

\section{Rong Xiao,}

Department of Molecular Biology and Biochemistry, Center of Advanced Biotechnology and Medicine, Rutgers, The State University of New Jersey, Piscataway, NJ 08854, USA

Department of Biochemistry, Robert Wood Johnson Medical School, UMDNJ, and Northeast Structural Genomics Consortium, Piscataway, NJ 08854, USA

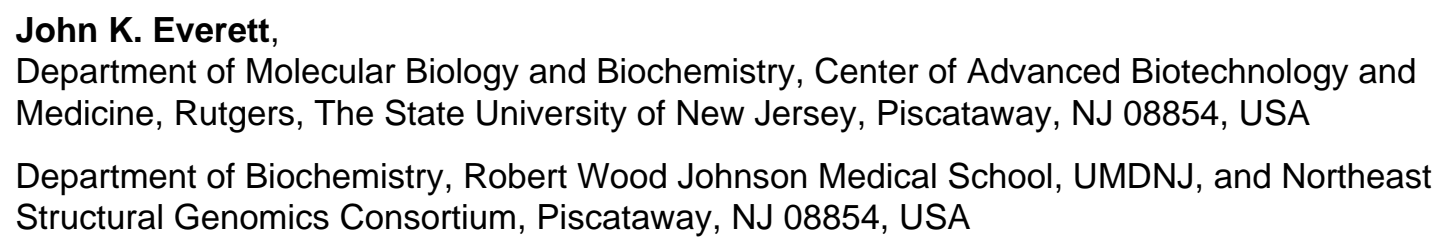

\footnotetext{
(c) Springer Science+Business Media B.V. 2011

Correspondence to: Thomas Szyperski.

Electronic supplementary material The online version of this article (doi:10.1007/s10969-011-9121-3) contains supplementary material, which is available to authorized users.
} 
Department of Biochemistry and Molecular Biophysics, Howard Hughes Medical Institute, Center for Computational Biology and Bioinformatics, Columbia University, New York, NY 10032, USA

Gaetano T. Montelione, and

Department of Molecular Biology and Biochemistry, Center of Advanced Biotechnology and Medicine, Rutgers, The State University of New Jersey, Piscataway, NJ 08854, USA

Department of Biochemistry, Robert Wood Johnson Medical School, UMDNJ, and Northeast Structural Genomics Consortium, Piscataway, NJ 08854, USA

\section{Thomas Szyperski}

Department of Chemistry, The State University of New York at Buffalo, and Northeast Structural Genomics Consortium, Buffalo, NY 14260, USA

\section{Abstract}

High-quality NMR structures of the homo-dimeric proteins Bvu3908 (69-residues in monomeric unit) from Bacteroides vulgatus and Bt2368 (74-residues) from Bacteroides thetaiotaomicron reveal the presence of winged helix-turn-helix (wHTH) motifs mediating tight complex formation. Such homo-dimer formation by winged HTH motifs is otherwise found only in two DNA-binding proteins with known structure: the C-terminal wHTH domain of transcriptional activator FadR from E. coli and protein TubR from B. thurigensis, which is involved in plasmid DNA segregation. However, the relative orientation of the wHTH motifs is different and residues involved in DNA-binding are not conserved in Bvu3908 and Bt2368. Hence, the proteins of the present study are not very likely to bind DNA, but are likely to exhibit a function that has thus far not been ascribed to homo-dimers formed by winged HTH motifs. The structures of Bvu3908 and Bt2368 are the first atomic resolution structures for PFAM family PF10771, a family of unknown function (DUF2582) currently containing 128 members.

\section{Keywords}

Bvu3908; Bt2368; PF10771; DUF2582; Winged helix-turn-helix; Structural genomics

\section{Introduction}

Bvu3908 (UniProtKB ID A6L747) and Bt2368 (Uni-ProtKB ID Q8A574) are 69- and 74residue proteins from Bacteroides vulgatus and Bacteroides thetaiotaomicron, respectively. The two proteins share $37 \%$ sequence identity (Fig. 1a) and belong to Pfam [1] protein family PF10771 of unknown function (DUF2582), which currently contains 128 members from eubacteria and archaeabacteria. Neither of the two proteins has significant sequence similarity to any protein with known structure. Bvu3908 and Bt2368 were selected by the Northeast Structural Genomics Consortium (NESG; http://www.nesg.org; target IDs BvR153 and BtR375, respectively) as part of a Protein Structure Initiative program aimed at determining 3D structures of novel proteins discovered by metagenomic sequencing of the human gut microbiome [2-4]. Here we report the high-quality solution NMR structures of Bvu3908 and Bt2368, which represent the first atomic resolution structures for PF10771.

\section{Materials and methods}

Bvu3908 and Bt2368 were cloned, expressed, and purified following protocols [5-7] established by the NESG (see Supplementary Material for details; http:// www.nmr2.buffalo.edu/nesg.wiki). The proteins included short C-terminal hexaHis tags (LEHHHHHH). The corresponding pET expression vectors (BvR153-21.1 and BtR375-21.2), have been deposited in the PSI Materials Repository (http://psimr.asu.edu/). 
[U- $\left.{ }^{13} \mathrm{C} ;{ }^{15} \mathrm{~N}\right]-$ and $\left[5 \%{ }^{13} \mathrm{C}\right.$; U_- $\left.{ }^{15} \mathrm{~N}\right]-$ labeled Bvu3908 NMR samples were prepared at $~ 1.1-$ $\mathrm{mM}$ concentration in a buffer containing $90 \% \mathrm{H}_{2} \mathrm{O} / 10 \% \mathrm{D}_{2} \mathrm{O}, 20 \mathrm{mM}$ ammonium acetate, $100 \mathrm{mM} \mathrm{NaCl}, 10 \mathrm{mM}$ DTT, $5 \mathrm{mM} \mathrm{CaCl}_{2}$, and $0.02 \% \mathrm{NaN}_{3}$ at $\mathrm{pH}$ 4.5, while Bt2368 NMR samples were prepared at $\sim 1.3 \mathrm{mM}$ concentration in a buffer containing $90 \% \mathrm{H}_{2} \mathrm{O} / 10 \%$ $\mathrm{D}_{2} \mathrm{O}, 20 \mathrm{mM}$ MES, $100 \mathrm{mM} \mathrm{NaCl}, 10 \mathrm{mM}$ DTT, $5 \mathrm{mM} \mathrm{CaCl}_{2}$, and $0.02 \% \mathrm{NaN}_{3}$ at $\mathrm{pH}$ 6.5. The $\left[5 \%{ }^{13} \mathrm{C}\right.$; U- $\left.{ }^{15} \mathrm{~N}\right]$-labeled samples enabled stereospecific assignment of the methyl groups of Val and Leu residues [8]. Isotropic overall rotational correlation times of about 8.0 and $8.4 \mathrm{~ns}$ were inferred for Bvu3908 and Bt2368, respectively, from ${ }^{15} \mathrm{~N}$ spin relaxation times, indicating that both proteins form homodimers in solution. This finding was confirmed by analytical gel-filtration (Agilent Technologies) followed by static light scattering (Supplementary Figs. S1, S2).

NMR data were acquired on Varian INOVA 600 and $750 \mathrm{MHz}$ spectrometers equipped with cryogenic ${ }^{1} \mathrm{H}\left\{{ }^{13} \mathrm{C},{ }^{15} \mathrm{~N}\right\}$ probes, a Bruker AVANCE $600 \mathrm{MHz}$ spectrometer equipped with a cryogenic ${ }^{1} \mathrm{H}\left\{{ }^{13} \mathrm{C},{ }^{15} \mathrm{~N}\right\}$ probe, and a Varian INOVA $500 \mathrm{MHz}$ spectrometer equipped with conventional ${ }^{1} \mathrm{H}\left\{{ }^{13} \mathrm{C},{ }^{15} \mathrm{~N}\right\}$ probe. Virtually complete sequence-specific ${ }^{1} \mathrm{H},{ }^{15} \mathrm{~N}$ and ${ }^{13} \mathrm{C}$ resonance assignments (Table 1; Supplementary Figs. S3, S4) were obtained from conventional triple-resonance NMR experiments (Supplementary Material) using the program AutoAssign 2.3.0 [9, 10], followed by manual assignment of side-chain resonances. Assignments were validated using the AVS software suite [11]. Backbone ${ }^{15} \mathrm{~N}-{ }^{1} \mathrm{H}$ residual dipolar couplings (RDCs) were measured for two alignment media in 2D $J$-modulated spectra (Supplementary Material). Chemical shifts, NOESY peak lists, RDCs, and timedomain data have been deposited in the BioMagResBank on 6/29/2010 (BMRB IDs: 17025 for Bvu3908; 17026 for Bt2368).

Structure calculations were performed using standardized methods of the NESG consortium $[12,13]$ using the program CYANA $3.0[14,15]$ based on ${ }^{1} \mathrm{H}-{ }^{1} \mathrm{H}$ NOE derived upper limit distance constraints, backbone dihedral angle constraints that were derived from chemical shifts using the program TALOS+ [16] for residues located in well-defined regular structure elements, and orientational constraints derived from polypeptide backbone ${ }^{15} \mathrm{~N}-{ }^{1} \mathrm{H}$ RDCs. Stereospecific assignment of methylene protons were performed using the GLOMSA module of CYANA and the final structure calculation was performed with CYANA followed by refinement of selected conformers in an 'explicit water bath' [17] using the program CNS 1.2 [18]. Validation of the resulting 20 refined conformers was performed with the Protein Structure Validation Software (PSVS) server 1.4 [19], and the agreement of structures and NOESY peak lists was verified using the AutoStructure/RPF 2.2.1 package [12].

The structural coverage provided by the structures of Bvu3908 and Bt2368 within PF10771 was calculated for the 87 unique sequences of this family using the program PSI-BLAST [20] with parameters published previously [21], i.e. E-value upper and lower cutoffs of $10^{-10}$ and 0.0005 , respectively.

A search for protein domains exhibiting structurally similar dimerization by a winged HTH motif was performed as follows. Structural neighbors of Bvu3908 were identified by searching a non-redundant structure database (60\% sequence identity cutoff) with the program SKAN [22] using a protein structural distance (PSD) cutoff [23] of 0.8. For each structural neighbor, we identified interfacial residues that aligned to the interfacial helix of Bvu3908 (residues 39-52) using the structure-based sequence alignment based on a heavyatom distance cutoff of $4.0 \AA$. 


\section{Results and discussion}

High-quality NMR structures of Bvu3908 and Bt2368 (Fig. 1b, c) were obtained (Table 1) and the coordinates were deposited in the Protein Data Bank [24] on 6/29/2010 (accession codes 2L01 and 2L02, respectively). The monomeric units of Bvu3908 and Bt2368 adopt a winged helix-turn-helix (wHTH) fold [25, 26], which consists of a three-stranded antiparallel $\beta$-sheet with topology $\mathrm{A}(\uparrow) \mathrm{C}(\downarrow) \mathrm{B}(\uparrow)$, and three $\alpha$-helices packed against one side of the sheet (Fig. 1d, e). The locations of the regular secondary structure elements are: $\beta$ strands A (residues 25-26 in Bvu3908/23-24 in Bt2368), B (55-60/52-57), C (63-67/6065), and a-helices I (4-19/3-19), II (27-34/25-31) and III (40-51/36-47), with a-helices II and III participating in the HTH motif. The subunits form tight binding homodimeric proteins resulting in buried surface areas of $1,800 \AA^{2}$ for Bvu3908 and 1,980 $\AA^{2}$ for Bt2368. The interfaces of the homodimers are formed primarily by hydrophobic side-chains (Leu 4, Ala 8, Phe 43, Leu 44, Leu 46, Trp 48, Leu 50 in Bvu3908; Val 6, Ile 25, Ala 40, Leu 41, Ala 47, Ile 54, Ile 61, Ile 63 in Bt2368). Five of the hydrophobic residues belong to a-helix III, which thus promotes dimerization by forming a coiled-coil like arrangement between the a-helices III of the two subunits. The monomeric units of Bvu3908 and Bt2368 are very similar, with the root mean square deviation (RMSD) calculated for the mean coordinates of the backbone heavy atoms $\mathrm{N}, \mathrm{C}^{\mathrm{a}}$ and $\mathrm{C}^{\prime}$ of regular secondary structure elements to be only $1.1 \AA$. The relative orientation of the monomeric units is, however, somewhat different as reflected by the corresponding RMSD of $2.9 \AA$ calculated for the homodimeric proteins. The major difference is observed for the relative orientations of a-helix II as well as the tip of the 'wing' comprised of the $\beta$-strands $\mathrm{B}$ and $\mathrm{C}$ and the intervening turn: in adjacent subunits these substructures are positioned closer in the Bt2368 homodimer which results in a more compact shape when compared with Bvu3908.

Helix-turn-helix motifs are characterized by the presence of two conserved sequence motifs in which polar residues ('p'), hydrophobic residues (' $h$ ') and small residues ('s') form a tripeptide segment with consensus signatures 'phs' in a-helix II and 'shs' in the turn connecting a-helices II and III [25]. In Bt2368, these segments are 'Glu 27-Leu 28-Ala 29' in helix II and 'Asn 32-Leu 33-Ser 34' in the turn. In Bt2368, deviations are observed from this consensus signature: in 'Gln 29-Ile30-Lys 31' and 'Lys35-Leu 36-Lys 37' small residues are replaced by Lys residues.

Both dimeric proteins exhibit quite similar conserved surface regions formed by residues located in a-helix I, the C-terminal segment of a-helix II, and the turn connecting this helix with $\beta$-strand B (Fig. 1a; Supplementary Fig. S5). Analysis of the structures using the programs ProFunc [27] and Mark-Us [28] revealed that these conserved surface regions contain three cavities, one centrally located at the interface and one located within each of the subunit. The central cavity is formed primarily by two conserved Trp and Arg residues (Trp 48 and Arg 51 in Bvu3908; Trp 45 and Arg 48 in Bt2368), and the other two cavities likewise involve conserved Trp and Arg residues along with conserved Gly, Glu and Lys residues (Gly 9, Gly 13, Trp 16, Trp 48, Arg 51, Glu 52 and Lys 54 in Bvy3908; Gly 7, Gly 11, Trp 14, Trp 45, Arg 48, Glu 49 and Lys 51 in Bt2368). The opposite face of both proteins shows high variability, with the exception of Lys 31 and Lys 35 of Bvu3908. The resulting electrostatic surface potential of the two proteins is thus of mixed positive and negative charge (with the opposite face being more positively charged in Bvu3908 and more negatively charged in Bt2368; Supplementary Fig. S6).

As expected for a structure comprising a HTH motif, a search of the PDB [24] for structurally similar proteins using the DALI server [29] yielded a very large number of structurally quite similar significant hits spanning a quasi-continuum of $Z$-scores $>4.0$ (i.e., more than 700 for both Bvu3908 and Bt2368). The highest $Z$-scores resulted for both 
proteins for the HTH motif of the ArsR-type DNA-binding domain of protein MJ1563 from Methanococcus janashii (PDB code 1KU9; Z-scores 9.4/10.3; RMSD of $\mathrm{C}^{\mathrm{a}}$ atoms: 2.9/2.4 $\AA$ A $64 / 63$ aligned residues with $17 \% / 11 \%$ sequence identity to Bvu3908/Bt2368). The vast majority of the remaining hits are, likewise as expected, nucleic acid binding proteins in which the 'recognition a-helix III' conveys nucleic acid sequence specific binding. In Bvu3908 and Bt2368 the a-helix III is buried in the interface of the homodimer. In stark contrast, a specific search for HTH domain structures exhibiting dimerization via a-helix III yielded only two proteins, FadR from E. coli[30, 31] (PDB codes 1E2X, 1H9G, 1H9T, 1HW1 and 1HW2) and TubR from B. thuringiensis [32] (PDB code 3M8E), both actually ranking in the lower half of the list of significant DALI hits. FadR is a transcriptional repressor/activatior involved in fatty acid metabolism and comprised of an N-terminal acylCoA binding domain and a C-terminal wHTH domain [30, 31]. TubR is a 104-residue protein encoded by the $B$. thuringiensis pBtoxis plasmid responsible for plasmid DNA segregation [32].

According to DALI, the monomeric units of Bvu3908/Bt2368 are structurally somewhat more similar to chain B of TubR (3M8E; $Z$-scores 5.9/6.6; RMSD of $\mathrm{C}^{a}$ atoms: 2.4/1.9 $\AA$; $60 / 60$ aligned residues with $13 \% / 15 \%$ sequence identity) than to chain $\mathrm{A}$ of FadR in its most similar structure (1HW1; $Z$-scores 4.7/5.3; RMSD of $\mathrm{C}^{\mathrm{a}}$ atoms: 2.7/2.7 $\AA$; 58/59 aligned residues with $14 \% / 14 \%$ sequence identity). However, the relative orientation of monomeric units in Bvu3908/Bt2368 is actually significantly more similar to the FadR than the TubR wHTH domain. Visual inspection reveals that the polypeptide backbone of the Bvu3908 dimer is overall structurally very similar to the one of FadR wHTH domain dimer, with the exception of the longer and more twisted 'wings' and a different orientation of a-helices I relative to the rest of the molecule (Supplementary Fig. S7A). The structure of the Bt2368 dimer exhibits the same deviations in its 'wings' and ahelices I, but exhibits, in addition, a different relative orientation of a-helices III at the dimer interface, coming along with a much closer location of a-helices II (Supplementary Fig. S7B). In contrast, the monomeric units of TubR are tilted significantly more relative to each other when compared with both Bvu3908 and Bt2368 (Supplementary Fig. S8), which is primarily due to the fact that ahelix I is much shorter in TubR than in Bvu3908 and Bt2368.

Both FadR and TubR wHTH domains were shown to bind to their specific double-stranded DNA regions in essentially the same 'non-canonical' manner, such that the tips of the ahelices III interact with the major groove of the DNA while the 'wings' are inserted into the adjacent minor grooves [30-32]. The DNA-binding interfaces of FadR and TubR form conserved positively charged bands on their surfaces (Supplementary Fig. S9). The dimer interfaces between FadR and TubR wHTH domains are formed mainly through interactions between a-helices III resulting in 'dumbbell' shapes of the dimers (Fig S9). The $\beta$-hairpin 'wings' of FadR and TubR are thus left as being exposed, allowing them to interact with the minor grooves of the DNA, while the sugar-phosphate backbone is accommodated in the protein grooves. In contrast, the $\beta$-hairpin 'wings' of Bvu3908 and Bt2368 form part of the homo-dimer interface. Furthermore, Bt2368 lacks a positively charged surface patch (Fig S6B), and though Bvu3908 does exhibit one (Fig S6A), it has a high sequence variability among Bvu3908 homologs, and runs nearly perpendicular to the bands in FadR and TubR. Of the nine residues in FadR that form the DNA-binding interface (Glu 34, Arg 35, Thr 44, Arg 45, Thr 46, Thr 47, Arg 49, Glu 50, His 65) none is conserved in Bvu3908 and Bt2368, and only Lys 28 of Bvu3908 and Arg 56 of Bt2368 can be considered equivalent to Arg 35 and His 65, respectively. The key basic residues of TubR required for interaction with DNA (Lys 43, Arg 74, Arg 77 and Lys 79) are not conserved in Bvu3908, and only Arg 74 matches Arg 56 in Bt2368. Taken together, it is very unlikely that Bt2368 binds to DNA and while this might be the case for Bvu3908, complex formation would have to be quite different from that observed for FadR and TubR. Hence, we conclude that the members of 
PF10771 function in way that has not yet been described for proteins forming homo-dimers via a winged HTH motif.

Finally, the novel structural leverage, that is, the number of new protein structures that can be reliably modeled, [21,33] provided by the structures of Bvu3908 and Bt2368 within PF10771 is 17 each. Since the modeling families for the two structures do no overlap, the two atomic resolution structures yield a total structural coverage of $39 \%$ for the 87 unique sequences of PF10771.

\section{Supplementary Material}

Refer to Web version on PubMed Central for supplementary material.

\section{Acknowledgments}

We thank D. Wang, C. Ciccosanti, K. Hamilton and S. Bhattacharya for helpful discussions and technical support. This work was supported by the National Institutes of Health, grant number: U54 GM094597 (T.S. and G.T.M.). While data acquisition was in progress, Prof. T. Szyperski was a member of the New York Structural Biology Center. The Center is a STAR center supported by the New York State Office of Science, Technology, and Academic Research.

\section{Abbreviations}

$\begin{array}{ll}\text { DSS } & \text { 4,4-Dimethyl-4-silapentane-1-sulfonate sodium salt } \\ \text { DTT } & \text { Dithiothreitol } \\ \text { HTH } & \text { Helix-turn-helix } \\ \text { MES } & \text { 2-(N-Morpholino)ethanesulfonic acid } \\ \text { NESG } & \text { Northeast Structural Genomics Consortium } \\ \text { NOE } & \text { Nuclear Overhauser effect } \\ \text { PDB } & \text { Protein Data Bank } \\ \text { RDC } & \text { Residual dipolar coupling } \\ \text { RMSD } & \text { Root mean square deviation } \\ \text { wHTH } & \text { Winged helix-turn-helix }\end{array}$

\section{References}

1. Finn RD, Mistry J, Tate J, Coggill P, Heger A, Pollington JE, Gavin OL, Gunasekaran P, Ceric G, Forslund K, Holm L, Sonnhammer ELL, Eddy SR, Bateman A. The Pfam protein families database. Nucleic Acids Res. 2010; 38:D211-D222. [PubMed: 19920124]

2. Xu J, Bjursell MK, Himrod J, Deng S, Carmichael LK, Chiang HC, Hooper LV, Gordon JI. A genomic view of the human-Bacteroides thetaiotaomicron symbiosis. Science. 2003; 299:20742076. [PubMed: 12663928]

3. Xu J, Mahowald MA, Ley RE, Lozupone CA, Hamady M, Martens EC, Henrissat B, Coutinho PM, Minx P, Latreille P, Cordum H, Van Brunt A, Kim K, Fulton RS, Fulton LA, Clifton SW, Wilson RK, Knight RD, Gordon JI. Evolution of symbiotic bacteria in the distal human intestine. PLoS Biol. 2007; 5:1574-1586.

4. Gill SR, Pop M, DeBoy RT, Eckburg PB, Turnbaugh PJ, Samuel BS, Gordon JI, Relman DA, Fraser-Liggett CM, Nelson KE. Metagenomic analysis of the human distal gut microbiome. Science. 2006; 312:1355-1359. [PubMed: 16741115]

5. Acton, TB.; Gunsalus, KC.; Xiao, R.; Ma, LC.; Aramini, J.; Baran, MC.; Chiang, YW.; Climent, T.; Cooper, B.; Denissova, NG.; Douglas, SM.; Everett, JK.; Ho, CK.; Macapagal, D.; Rajan, PK.; 
Shastry, R.; Shih, LY.; Swapna, GVT.; Wilson, M.; Wu, M.; Gerstein, M.; Inouye, M.; Hunt, JF.; Montelione, GT. Nuclear magnetic resonance of biological macromolecules, Part. C. In: James, TL., editor. Methods in enzymology. Vol. vol 394. San Diego: Elsevier; 2005. p. 210-243.

6. Xiao R, Anderson S, Aramini J, Belote R, Buchwald WA, Ciccosanti C, Conover K, Everett JK, Hamilton K, Huang YJ, Janjua H, Jiang M, Kornhaber GJ, Lee DY, Locke JY, Ma LC, Maglaqui M, Mao L, Mitra S, Patel D, Rossi P, Sahdev S, Sharma S, Shastry R, Swapna GVT, Tong SN, Wang DY, Wang HA, Zhao L, Montelione GT, Acton TB. The high-throughput protein sample production platform of the Northeast Structural Genomics Consortium. J Struct Biol. 2010; 172:2133. [PubMed: 20688167]

7. Acton, TB.; Xiao, R.; Anderson, S.; Aramini, J.; Buchwald, WA.; Ciccosanti, C.; Conover, K.; Everett, J.; Hamilton, K.; Huang, YJ.; Janjua, H.; Kornhaber, G.; Lau, J.; Lee, DY.; Liu, GH.; Maglaqui, M.; Ma, LC.; Mao, L.; Patel, D.; Rossi, P.; Sahdev, S.; Shastry, R.; Swapna, GVT.; Tang, YF.; Tong, SC.; Wang, DY.; Wang, H.; Zhao, L.; Montelione, GT. Fragment-based drug design: tools, practical approaches, and examples. In: Kuo, LC., editor. Methods in enzymology. Vol. vol 493. San Diego: Elsevier; 2011. p. 21-60.

8. Neri D, Szyperski T, Otting G, Senn H, Wuthrich K. Stereospecific nuclear magnetic resonance assignments of the methyl groups of valine and leucine in the DNA-binding domain of the 434 repressor by biosynthetically directed fractional 13C labeling. Biochemistry. 1989; 28:7510-7516. [PubMed: 2692701]

9. Zimmerman DE, Kulikowski CA, Huang YP, Feng WQ, Tashiro M, Shimotakahara S, Chien CY, Powers R, Montelione GT. Automated analysis of protein NMR assignments using methods from artificial intelligence. J Mol Biol. 1997; 269:592-610. [PubMed: 9217263]

10. Moseley, HNB.; Monleon, D.; Montelione, GT. Nuclear magnetic resonance of biological macromolecules, Pt B. In: James, TL.; Dötsch, V.; Schmitz, U., editors. Methods in enzymology. Vol. vol 339. San Diego: Elsevier; 2001. p. 91-108.

11. Moseley HNB, Sahota G, Montelione GT. Assignment validation software suite for the evaluation and presentation of protein resonance assignment data. J Biomol NMR. 2004; 28:341-355. [PubMed: 14872126]

12. Huang YJ, Powers R, Montelione GT. Protein NMR recall, precision, and F-measure scores (RPF scores): structure quality assessment measures based on information retrieval statistics. $\mathrm{J}$ Am Chem Soc. 2005; 127:1665-1674. [PubMed: 15701001]

13. Liu GH, Shen Y, Atreya HS, Parish D, Shao Y, Sukumaran DK, Xiao R, Yee A, Lemak A, Bhattacharya A, Acton TA, Arrow-smith CH, Montelione GT, Szyperski T. NMR data collection and analysis protocol for high-throughput protein structure determination. Proc Natl Acad Sci USA. 2005; 102:10487-10492. [PubMed: 16027363]

14. Guntert P, Mumenthaler C, Wuthrich K. Torsion angle dynamics for NMR structure calculation with the new program DYANA. J Mol Biol. 1997; 273:283-298. [PubMed: 9367762]

15. Herrmann T, Guntert P, Wuthrich K. Protein NMR structure determination with automated NOE assignment using the new software CANDID and the torsion angle dynamics algorithm DYANA. J Mol Biol. 2002; 319:209-227. [PubMed: 12051947]

16. Cornilescu G, Delaglio F, Bax A. Protein backbone angle restraints from searching a database for chemical shift and sequence homology. J Biomol NMR. 1999; 13:289-302. [PubMed: 10212987]

17. Linge JP, Williams MA, Spronk C, Bonvin A, Nilges M. Refinement of protein structures in explicit solvent. Proteins. 2003; 50:496-506. [PubMed: 12557191]

18. Brunger AT, Adams PD, Clore GM, DeLano WL, Gros P, Grosse-Kunstleve RW, Jiang JS, Kuszewski J, Nilges M, Pannu NS, Read RJ, Rice LM, Simonson T, Warren GL. Crystallography \& NMR system: a new software suite for macromolecular structure determination. Acta Crystallogr Sect D Biol Crystallogr. 1998; 54:905-921. [PubMed: 9757107]

19. Bhattacharya A, Tejero R, Montelione GT. Evaluating protein structures determined by structural genomics consortia. Proteins Struct Funct Bioinf. 2007; 66:778-795.

20. Altschul SF, Madden TL, Schaffer AA, Zhang JH, Zhang Z, Miller W, Lipman DJ. Gapped BLAST and PSI-BLAST: a new generation of protein database search programs. Nucleic Acids Res. 1997; 25:3389-3402. [PubMed: 9254694] 
21. Liu JF, Montelione GT, Rost B. Novel leverage of structural genomics. Nat Biotechnol. 2007; 25:850-853.

22. Petrey, D.; Honig, B. Macromolecular crystallography, Pt D. In: Carter, CW.; Sweet, RM., editors. Methods in enzymology. Vol. vol 374. San Diego: Elsevier; 2003. p. 492-509.

23. Yang AS, Honig B. An integrated approach to the analysis and modeling of protein sequences and structures. I. Protein structural alignment and a quantitative measure for protein structural distance. J Mol Biol. 2000; 301:665-678. [PubMed: 10966776]

24. Berman HM, Westbrook J, Feng Z, Gilliland G, Bhat TN, Weissig H, Shindyalov IN, Bourne PE. The Protein Data Bank. Nucleic Acids Res. 2000; 28:235-242. [PubMed: 10592235]

25. Aravind L, Anantharaman V, Balaji S, Babu MM, Iyer LM. The many faces of the helix-turn-helix domain: transcription regulation and beyond. FEMS Microbiol Rev. 2005; 29:231-262. [PubMed: 15808743]

26. Gajiwala KS, Burley SK. Winged helix proteins. Curr Opin Struct Biol. 2000; 10:110-116. [PubMed: 10679470]

27. Laskowski RA, Watson JD, Thornton JM. ProFunc: a server for predicting protein function from 3D structure. Nucleic Acids Res. 2005; 33:W89-W93. [PubMed: 15980588]

28. Petrey D, Fischer M, Honig B. Structural relationships among proteins with different global topologies and their implications for function annotation strategies. Proc Natl Acad Sci USA. 2009; 106:17377-17382. [PubMed: 19805138]

29. Holm L, Sander C. Dali-a network tool for protein structure comparison. Trends Biochem Sci. 1995; 20:478-480. [PubMed: 8578593]

30. van Aalten DMF, Dirusso CC, Knudsen J. The structural basis of acyl coenzyme A-dependent regulation of the transcription factor FadR. EMBO J. 2001; 20:2041-2050. [PubMed: 11296236]

31. Xu YB, Heath RJ, Li ZM, Rock CO, White SW. The FadR DNA complex-transcriptional control of fatty acid metabolism in Escherichia coli. J Biol Chem. 2001; 276:17373-17379. [PubMed: 11279025]

32. Ni LS, Xu WJ, Kumaraswami M, Schumacher MA. Plasmid protein TubR uses a distinct mode of HTH-DNA binding and recruits the prokaryotic tubulin homolog TubZ to effect DNA partition. Proc Natl Acad Sci USA. 2010; 107:11763-11768. [PubMed: 20534443]

33. Nair R, Liu J, Soong T-T, Acton TB, Everett JK, Kouranov A, Fiser A, Godzik A, Jaroszewski L, Orengo C, Montelione GT, Rost B. Structural genomics is the largest contributor of novel structural leverage. J Struct Funct Genomics. 2009; 10:181-191. [PubMed: 19194785]

34. Laskowski RA, Rullmann JAC, MacArthur MW, Kaptein R, Thornton JM. AQUA and PROCHECK-NMR: programs for checking the quality of protein structures solved by NMR. $J$ Biomol NMR. 1996; 8:477-486. [PubMed: 9008363]

35. Chen VB, Arendall WB, Headd JJ, Keedy DA, Immormino RM, Kapral GJ, Murray LW, Richardson JS, Richardson DC. MolProbity: all-atom structure validation for macromolecular crystallography. Acta Crystallogr Sect D Biol Crystallogr. 2010; 66:12-21. [PubMed: 20057044]

36. Luthy R, Bowie JU, Eisenberg D. Assessment of protein models with 3-dimensional profiles. Nature. 1992; 356:83-85. [PubMed: 1538787]

37. Sippl MJ. Recognition of errors in 3-dimensional structures of proteins. Proteins. 1993; 17:355362. [PubMed: 8108378] 
A

Bvu3908

Bt2368

A7M526 BACOV

A5ZLA4_9BACE

Q64T73_BACFR

Q8A6Q7_BACTN
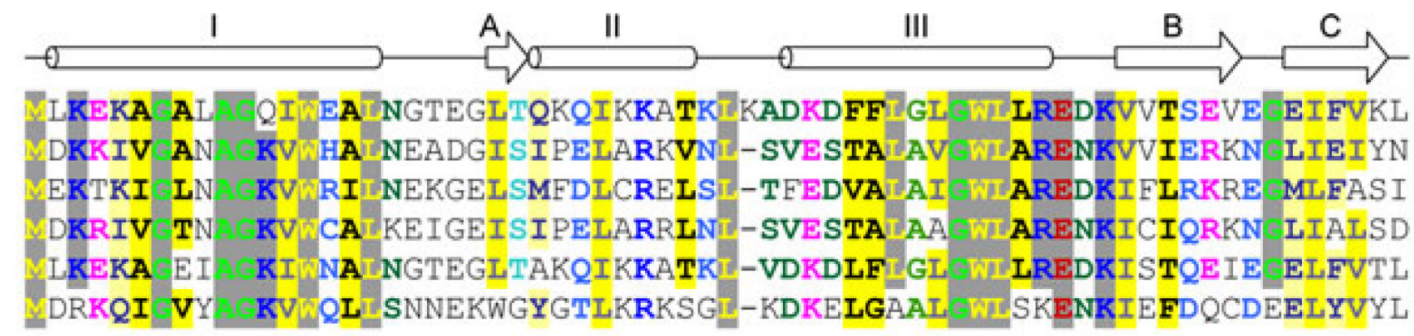

B
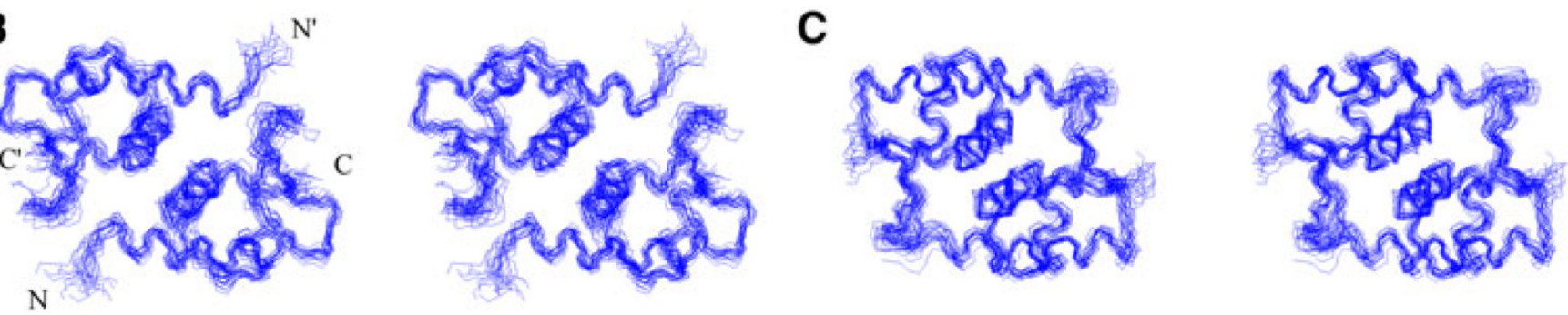

D
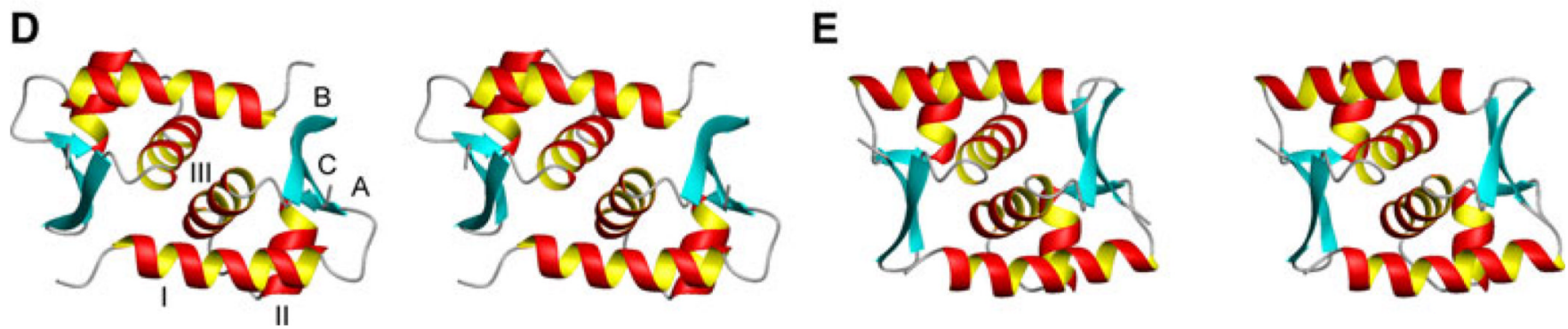

Fig. 1.

a Multiple sequence alignment of a representative subset of members of PF10771, including Bvu3908, Bt2368 along with homologues from Bacteroides ovatus, Bacteroides caccae, Bacteroides fragilis and Bacteroides thetaiotaomicron. The location of the regular secondary structure elements observed in Bvu3908 are indicated above the alignment. The figure was generated with the program Chroma v1.0 using the default color scheme: negative (red), positive (dark blue), charged (magenta), polar (light blue), Ser/Thr (cyan), tiny (light green), small (dark green), aliphatic (gray on yellow), aromatic (blue on yellow), big (violet on pale yellow), hydrophobic (black on yellow). b Stereoview of the 20 conformers representing the solution structure of Bvu3908 obtained after superposition of the $\mathrm{C}^{\mathrm{a}}$ atoms of the regular secondary structure elements for minimal root-mean-square deviation (RMSD). Residues 70-77 of the disordered C-terminal polypeptide segment were omitted for clarity, and the Nand C-termini are labeled as "N" and "C". c Same as in B but for Bt2368. Residues 68-75 of the disordered C-terminal polypeptide segment were omitted for clarity. d Stereoview of a ribbon diagram of the lowest-energy conformer of Bvu3908: a-helices are shown in red and yellow, $\beta$-strands are depicted in cyan, other polypeptide segments are in gray. $\mathbf{e}$ Same as in d for $\mathrm{Bt} 2368$ 
Table 1

Statistics for structures of Bvu3908 and Bt2368

\begin{tabular}{|c|c|c|}
\hline & Bvu3908 & Bt2368 \\
\hline \multicolumn{3}{|l|}{ Completeness of resonance assignments ${ }^{a}(\%)$} \\
\hline Backbone/side-chain & $99 / 99$ & $100 / 95$ \\
\hline \multicolumn{3}{|l|}{ Completeness of stereospecific assignments $b$} \\
\hline Val and Leu isopropyl/ $/{ }^{\beta} \mathrm{CH}_{2} /{ }^{a} \mathrm{CH}_{2}$ of Gly & $90 / 0 / 8$ & $92 / 6 / 40$ \\
\hline \multicolumn{3}{|l|}{ Conformation-restricting distance constraints ${ }^{c}$} \\
\hline Intraresidue $[i=j]$ & 248 & 199 \\
\hline Sequential $[|i-\lambda|=1]$ & 297 & 270 \\
\hline Medium range $[1<|i-\jmath|<5]$ & 313 & 293 \\
\hline Long range $[|i-\lambda| \geq 5]$ & 360 & 288 \\
\hline Intermolecular & 21 & 20 \\
\hline Total & 1,218 & 1,050 \\
\hline Dihedral angle constraints $(\varphi / \psi)$ & $56 / 56$ & $62 / 62$ \\
\hline Residual dipolar coupling constraints $\left({ }^{15} \mathrm{~N}-{ }^{1} \mathrm{H}\right)$ & 97 & 94 \\
\hline Distance constraints per residue (of those, long-range) & $18.5(5.0)$ & $15.4(3.8)$ \\
\hline CYANA target function $\left(\AA^{2}\right)$ & $1.02 \pm 0.21$ & $1.88 \pm 0.21$ \\
\hline \multicolumn{3}{|c|}{ Average number of distance constraint violations per conformer } \\
\hline $0.2-0.5 \AA$ & 5.8 & 5.6 \\
\hline$>0.5 \AA$ & 0.0 & 0.1 \\
\hline \multicolumn{3}{|c|}{ Average number of dihedral angle constraint violations per conformer } \\
\hline$>10^{\circ}$ & 0.0 & 0.0 \\
\hline \multicolumn{3}{|l|}{ Average RMSD from mean coordinates $(\AA)$} \\
\hline Backbone heavy atoms (all heavy atoms) ${ }^{d}$ & $0.8(1.1)$ & $0.6(1.1)$ \\
\hline Backbone heavy atoms (all heavy atoms) ${ }^{e}$ & $0.8(1.2)$ & $0.7(1.1)$ \\
\hline \multicolumn{3}{|l|}{ Global quality scores ${ }^{\mathcal{c}}$ (raw/Z-score) } \\
\hline PROCHECK [34] G-factor ( $\varphi$ and $\psi$ ) & $0.19 / 1.06$ & $0.19 / 1.06$ \\
\hline PROCHECK [34] G-factor (all dihedral angles) & $0.11 / 0.65$ & $0.11 / 0.65$ \\
\hline Molprobity [35] clash score & $12.58 /-0.63$ & $12.34 /-0.59$ \\
\hline Verify3D [36] & $0.42 /-0.64$ & $0.34 /-1.93$ \\
\hline ProsaII [37] & $0.76 / 0.45$ & $0.65 / 0.00$ \\
\hline \multicolumn{3}{|l|}{ RPF scores [12] } \\
\hline Recall/precision/F-measure & $0.98 / 0.84 / 0.90$ & $0.98 / 0.83 / 0.90$ \\
\hline DP-score & 0.87 & 0.83 \\
\hline \multicolumn{3}{|l|}{ Molprobity [35] Ramachandran summary ${ }^{e_{(\%)}}$} \\
\hline Most favored regions & 99.6 & 98.8 \\
\hline Additionally allowed regions & 0.4 & 1.2 \\
\hline Disallowed regions & 0.0 & 0.0 \\
\hline
\end{tabular}

${ }^{a}$ Calculated with AVS suite [11] excluding eight non-native residues of the C-terminal tag, N-terminal and Lys and Arg side chain amino groups, hydroxyl of Ser, Thr and Tyr, carboxyls of Asp and Glu, and non-protonated aromatic carbons 
${ }^{b}$ Relative to pairs with non-degenerate chemical shifts

${ }^{c}$ Calculated with PSVS 1.4 [19]

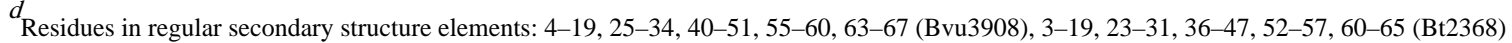

erdered residues: 3-35, 36-67 (Bvu3908), 3-65 (Bt2368) 